\title{
Impact of Canagliflozin Treatment on Health-Related Quality of Life among People with Type 2 Diabetes Mellitus: A Pooled Analysis of Patient-Reported Outcomes from Randomized Controlled Trials
}

\author{
Jennifer Cai ${ }^{1}$ (D) Linda M. Delahanty ${ }^{2} \cdot$ Sydney Akapame $^{3} \cdot$ April Slee $^{3}$. \\ Shana Traina ${ }^{4}$
}

Published online: 8 January 2018

(c) The Author(s) 2018. This article is an open access publication

\begin{abstract}
Background Evidence from patient-reported outcomes in clinical trials may explain health-related behaviors observed in the real world.

Objective The purpose of this analysis was to evaluate the effect of treatment with canagliflozin, a sodium glucose cotransporter 2 inhibitor, compared with placebo or sitagliptin on health-related quality-of-life outcomes in participants with type 2 diabetes mellitus from the clinical development program.

Methods Patient-reported outcomes data from four randomized controlled trials of canagliflozin $(n=2536)$ were pooled and analyzed to evaluate participants' interest in continuing study medication; satisfaction with weight; and physical, mental, and emotional health after 26-52 weeks of treatment with canagliflozin vs. placebo or sitagliptin.

Results Upon trial completion, participants treated with canagliflozin were more likely to express interest in continuing study medication than participants treated with placebo or sitagliptin [odds ratio (95\% confidence interval) of $1.54(1.19-1.99) ; p=0.001]$. Those treated with canagliflozin were also more likely to be satisfied with their
\end{abstract}

Electronic supplementary material The online version of this article (https://doi.org/10.1007/s40271-017-0290-4) contains supplementary material, which is available to authorized users.

Jennifer Cai

Jcai22@its.jnj.com

1 Janssen Scientific Affairs, LLC, 1125 Trenton-Harbourton Rd, Titusville, NJ 08560, USA

2 Massachusetts General Hospital Diabetes Center and Harvard Medical School, Boston, MA, USA

3 Axio Research, Seattle, WA, USA

4 Janssen Global Services, LLC, Raritan, NJ, USA weight and report favorable outcomes (score improvement or maintenance of good scores) related to physical and emotional health.

Conclusions The results of this pooled analysis suggest that people with type 2 diabetes mellitus treated with canagliflozin generally had positive experiences with treatment and improvements in health-related quality of life. Future research is needed to determine if these improvements result in improved type 2 diabetes mellitus management and treatment adherence.

ClinicalTrials.gov identifiers NCT01106625, NCT01106677, NCT01137812, NCT02025907.

\section{Key Points}

A higher proportion of participants with type 2 diabetes mellitus treated with canagliflozin reported interest in continuing treatment upon study completion compared with participants who received other treatments (placebo or sitagliptin).

Participants treated with canagliflozin were also more likely to be satisfied with their weight and physical and emotional health, demonstrating improved health-related quality of life compared with placebo or sitagliptin.

Further research is needed to determine if these positive outcomes will lead to improved disease management behaviors and treatment adherence among people with type 2 diabetes mellitus receiving canagliflozin. 


\section{Introduction}

Management of type 2 diabetes mellitus (T2DM) can be challenging for patients and their healthcare providers given the complexity of its pathophysiology and associated co-morbidities [1]. One obstacle facing physicians is that treatment decisions should fulfill the triple aim of improving the patient care experience and quality of care while containing costs [2]. Patient-centered care is central to T2DM management, with guidelines recommending that glycemic targets and treatment regimens be tailored to individual characteristics and preferences [1]. Additionally, there is an emphasis on creating and using tools for shared decision making in the treatment of T2DM [3]. There has been a gradual shift toward inclusion of patient-relevant endpoints in trials of new T2DM medications because patients are increasingly responsible for disease management decisions [4, 5]. Patient-reported outcome (PRO) instruments are used to capture endpoints that can only be measured from the patient's perspective [6]. Patient-reported outcomes can be used to describe the impact of treatment on patient perceptions of health; importantly, a patient's perception of their own health can influence their performance of self-care behaviors, including medication adherence and weight management via healthy eating and being active [7]. Type 2 diabetes mellitus is associated with worse health-related quality of life (HRQoL), and HRQoL is even worse when complications develop or comorbidities exist [8,9]. For patients with T2DM, the degree to which they are satisfied with their health and levels of HRQoL is associated with engagement in healthy self-care behaviors, which have been empirically linked to lower healthcare costs [10].

Canagliflozin, a sodium glucose co-transporter 2 inhibitor, has demonstrated improvements in glycemic control, with the added benefits of weight loss and blood pressure reduction, and a favorable tolerability profile across a broad range of people with T2DM in clinical studies compared with placebo as well as the active comparators sitagliptin and glimepiride [11]. Canagliflozin has also demonstrated consistent improvements in glycemic control compared with other antihyperglycemic agents (AHAs), including dipeptidyl peptidase-4 inhibitors, in real-world settings $[12,13]$. Patient-reported outcome instruments were used to describe satisfaction with health and HRQoL in several clinical studies of canagliflozin [14-18]. Analyses based on PRO data from these studies demonstrated that weight loss of $\sim 2.3 \mathrm{~kg}$ (the average amount seen with canagliflozin) was associated with significantly improved weight-related quality of life and health satisfaction [19], and that the amount and patterns of weight loss most commonly seen with canagliflozin had a significant positive impact on the performance of diabetes self-care behaviors [7]. Because individual trials were powered for clinical endpoints and thus not specifically powered to detect treatment differences in PRO results with canagliflozin vs. comparators, this analysis used pooled data from four clinical trials to summarize the impact of canagliflozin on patient experience with treatment, including willingness to continue treatment at the end of the studies. This analysis also examined changes in HRQoL and captured the impact of canagliflozin treatment on patients' satisfaction with weight, overall health, and physical and mental functioning.

\section{Methods}

\subsection{Analysis Set}

A total of five phase III and IV randomized, double-blind, global canagliflozin clinical trials included PRO measurements using a variety of instruments. To maximize statistical power for this analysis, PRO data were pooled from the four trials that collected data for the Current Health Satisfaction Questionnaire (CHES-Q), which was the most commonly used PRO instrument across studies: add-on to metformin vs. placebo/sitagliptin (Study 1; ClinicalTrials.gov identifier: NCT01106677) [14], add-on to metformin plus sulfonylurea vs. placebo (Study 2; NCT01106625) [15], add-on to metformin plus sulfonylurea vs. sitagliptin (Study 3; NCT01137812) [16], and add-on to metformin plus sitagliptin vs. placebo (Study 4; NCT02025907) [17]. The main objective of this analysis was to determine interest in continuing the study drug at the end of each study [week 52 (Studies 1-3) or week 26 (Study 4)]. Additional assessments included satisfaction with weight, physical function, and mental and emotional health at week 26 (common time point among all four studies). An overview of the studies, PRO instruments, and timing of measures included in this analysis is shown in Table 1 of the Electronic Supplementary Material (ESM).

\subsection{Outcomes}

\subsubsection{Interest in Continuing Study Medication}

At the end of the study (week 52 for Studies 1-3 and week 26 for Study 4), interest in continuing study medication was analyzed; participants, who remained blinded to their treatment group, were asked to rate their level of interest in continuing the study drug on a 7-point Likert scale: 1 ("Very Disinterested"), 2 ("Disinterested"), 3 ("Somewhat Disinterested"), 4 ("Neutral"), 5 ("Somewhat Interested"), 6 ("Interested"), and 7 ("Very Interested"). 
Table 1 Patient-reported outcome instrument scores and favorable outcome definitions

\begin{tabular}{|c|c|c|c|c|c|c|}
\hline \multirow[t]{2}{*}{ Instrument } & \multirow[t]{2}{*}{ Item or domain } & \multirow[t]{2}{*}{ Range $^{a}$} & \multirow[t]{2}{*}{ MCID } & \multicolumn{3}{|c|}{ Favorable outcome definitions } \\
\hline & & & & Maintenance & Improvement & $\begin{array}{l}\text { Interest in continuing } \\
\text { study medication }\end{array}$ \\
\hline $\begin{array}{l}\text { Interest in continuing study } \\
\text { medication }\end{array}$ & & $1-7$ & - & - & - & $\mathrm{FU} \geq 5$ \\
\hline \multirow[t]{3}{*}{ CHES-Q } & $\begin{array}{l}\text { Satisfaction with body } \\
\text { weight (item 1) }\end{array}$ & $1-7$ & 1 & $\begin{array}{l}\geq 5 \text { at } \mathrm{BL} \text { and } \\
\mathrm{FU}\end{array}$ & $\mathrm{FU} \geq \mathrm{BL}+\mathrm{MCID}$ & - \\
\hline & $\begin{array}{l}\text { Satisfaction with physical } \\
\text { health }\end{array}$ & $1-7$ & 0.17 & $\begin{array}{l}\geq 5 \text { at } \mathrm{BL} \text { and } \\
\mathrm{FU}\end{array}$ & $\mathrm{FU} \geq \mathrm{BL}+\mathrm{MCID}$ & - \\
\hline & $\begin{array}{l}\text { Satisfaction with emotional } \\
\text { health }\end{array}$ & $1-7$ & 0.33 & $\begin{array}{l}\geq 5 \text { at } \mathrm{BL} \text { and } \\
\mathrm{FU}\end{array}$ & $\mathrm{FU} \geq \mathrm{BL}+\mathrm{MCID}$ & - \\
\hline IWQoL-Lite & Total score & $0-100$ & 7.7 & $\begin{array}{l}\geq 92.3 \text { at } \mathrm{BL} \\
\text { and } \mathrm{FU}\end{array}$ & $\mathrm{FU} \geq \mathrm{BL}+\mathrm{MCID}$ & - \\
\hline \multirow[t]{2}{*}{ SF-36 } & $\begin{array}{l}\text { Physical component } \\
\text { summary score }\end{array}$ & $0-100$ & 1 & - & $\mathrm{FU} \geq \mathrm{BL}+\mathrm{MCID}$ & - \\
\hline & $\begin{array}{l}\text { Mental component } \\
\text { summary score }\end{array}$ & $0-100$ & 1 & - & $\mathrm{FU} \geq \mathrm{BL}+\mathrm{MCID}$ & - \\
\hline
\end{tabular}

$B L$ baseline, $C H E S-Q$ Current Health Satisfaction Questionnaire, FU follow-up, IWQoL-Lite Impact of Weight on Quality of Life-Lite, $M C I D$ minimal clinically important difference, $S F-36$ Short Form-36

${ }^{\mathrm{a}}$ For interest in continuing study medication and CHES-Q, 1 is the lowest possible score and 7 is the highest possible score. For IWQoL-Lite, 0 is the lowest possible score and 100 is the highest possible score. For SF-36, the raw score range is from 0 (lowest) to 100 (highest), and the range of normalized scores varies by study

Answers were dichotomized such that participants with scores of $\geq 5$ (positive responses) were considered to be interested and scores of $<5$ (negative or neutral responses) were considered to be not interested (Table 1).

\subsubsection{Patient-Reported Outcome Instruments and Minimal Clinically Important Differences}

An overview of PRO instruments analyzed in this study, favorable outcomes, and minimal clinically important differences (MCIDs) is shown in Table 1. Note that the MCID is a threshold designed to represent the smallest change in an outcome that a patient would perceive to be clinically important.

CHES-Q [20, 21] was used to measure diabetes-related health satisfaction in all four studies. Weight satisfaction was assessed using CHES-Q item 1 ("I am satisfied with my current body weight."). Participants responded on a 7-point Likert scale: 1 ("Strongly Disagree"), 2 ("Disagree"), 3 ("Somewhat Disagree"), 4 ("Neutral"), 5 ("Somewhat Agree"), 6 ("Agree"), and 7 ("Strongly Agree"). The CHES-Q physical domain consists of six items related to satisfaction with physical health: satisfaction with weight, energy, appetite, ability to do physical activities, sleep, and current health; scores are assessed on a 7-point scale (range 1-7), with higher scores indicating a greater degree of agreement with the statements. The CHES-Q emotional domain consists of three items related to satisfaction with emotional health: social interactions, attitude toward diabetes, and mood; scores are assessed on a 7-point scale (range 1-7), with higher scores indicating a greater degree of agreement with the statements. A change of 1 (corresponding to 1 category change) is considered to be clinically meaningful for CHES-Q item 1 , a change of 0.17 (equivalent to 1 of 6 on the 'average score scale', reflecting a net satisfaction improvement in at least one item) is considered to be clinically meaningful for the CHES-Q physical domain, and a change of 0.33 (equivalent to 1 of 3 on the 'average score scale', reflecting a net satisfaction improvement in at least one item) is considered to be clinically meaningful for the CHES-Q emotional domain [20, 22].

The Impact of Weight on Quality of Life-Lite (IWQoLLite) questionnaire was used to assess participants' perception of how weight affects day-to-day life in Studies 1-3 [23]; scores range from 0 to 100 , with higher scores indicating less burden of weight on quality of life. Total score was used in this analysis as it comprises elements of the five domain scores (physical function, self-esteem, sexual life, public distress, and work). For the IWQoL-Lite questionnaire, a change in total score of $\geq 7.7$ points is considered to be clinically meaningful [24].

The Short Form-36 (SF-36) questionnaire was used to capture changes in general physical and mental/emotional health in Studies 1-3; raw scores range from 0 to 100, with higher scores indicating better HRQoL. SF-36 scores were 
normalized prior to statistical analysis. Typically, improvement of at least 2-4 points is considered to be the MCID for the SF-36 questionnaire; however, for patients with T2DM, a 1-point reduction in physical function, general health or physical component summary scales has been associated with increased mortality risk [25], thus 1 point was used as the MCID for this analysis.

\subsubsection{Definitions of Favorable Outcomes}

Least squares (LS) mean changes from baseline are more statistically powerful than dichotomous outcomes; however, LS mean changes do not capture the proportion of participants who experienced improvement. Furthermore, participants with scores near the instrument ceiling at baseline have little room for improvement (though they can deteriorate). Because many participants in the canagliflozin studies had good PRO scores at baseline (i.e., within the MCID of the highest possible value of scores), a favorable outcome was defined for each PRO instrument as either maintenance of good scores or improvement in PRO scores in this analysis. Details on the definition of favorable outcomes for each PRO instrument can be found in Table 1.

\subsection{Statistical Analyses}

Interest in continuing study medication was evaluated using logistic regression models that included treatment group, baseline PRO score, and study. Least squares mean change from baseline to week 26 in PRO measures was assessed using an analysis of covariance (ANCOVA) with the treatment group as a fixed covariate and adjustments for baseline PRO score and study.

Improvement from baseline to week 26 in PRO instruments was evaluated using logistic regression models that included treatment group, baseline PRO score, and study. Odds ratios (ORs) and 95\% confidence intervals (CIs) were estimated for the proportions of people with any change in PRO measures. Comparisons were made between canagliflozin (100- and 300-mg doses were pooled because PRO results in the individual trials were similar between doses) and comparators (pooled placebo/sitagliptin) at week 26. Statistical significance was determined using a $p$ value of 0.05 . Based on prior examination of PRO results in trials of canagliflozin, it was expected that weight would be the largest driver of change in PRO scores [7, 19]; thus, data for placebo and sitagliptin were pooled for this analysis as they are generally considered to be weight neutral (in the current studies, placebo and sitagliptin provided changes in body weight of -1.6 to $0.1 \mathrm{~kg}$ [14-17]).

\subsection{Sensitivity Analyses}

Sensitivity analyses were performed for all regression models that adjusted for sex and baseline body mass index to determine whether the inclusion of these covariates impacted results. Sensitivity analyses were also performed using week 52 data for the three studies with data at this time point. Of note, Studies 1 and 2 were designed as 26-week studies with 26-week extension periods, and many participants did not participate in the extension phases for a range of reasons, such as the patient's decision to not begin rescue insulin or withdrawal of consent. Therefore, approximately one-third of participants in these studies did not have data at week 52. Study 4 was designed as a 26-week study with no extension phase and thus was not included in this sensitivity analysis. These models have lower power to detect treatment effects but were included to explore the robustness of the response.

\section{Results}

\subsection{Participants}

Trial design and participant characteristics from the four individual trials are shown in Table 2. A total of 1434 participants treated with canagliflozin and 1102 participants treated with placebo or sitagliptin who completed 26 or 52 weeks of treatment and had available PRO data were included in this pooled analysis. Completion of PRO instruments was $>81 \%$ among participants in the intent-totreat study populations, and was $>93 \%$ for participants who completed 26 weeks in Studies 1 and 2; therefore, the subset of participants with PRO data was generally representative of the overall pooled population. Participant characteristics and baseline PRO scores were generally balanced between the canagliflozin and placebo/sitagliptin groups in the pooled population (Table 3). Participants in the pooled canagliflozin group lost an average of $3.22 \mathrm{~kg}$ [standard deviation (SD) 3.75] at the end of the study compared with $0.86 \mathrm{~kg}$ (SD 3.40) in the placebo/sitagliptin groups.

\subsection{Regression Analyses}

Regression analyses were used to examine the impact of baseline value and individual study on PRO outcomes. Baseline PRO values were statistically significant predictors of the PRO outcome of interest. This finding supports the notion of ceiling effects in these PRO instruments (i.e., patients with higher baseline PRO scores were likely to maintain higher scores). Study was also a statistically significant predictor of PRO outcomes in most models, 
Table 2 Trial design and patient characteristics by individual trial

\begin{tabular}{|c|c|c|c|c|}
\hline & Study 1 [14] & Study 2 [15] & Study 3 [16] & Study 4 [17] \\
\hline Comparator & Sitagliptin and placebo & Placebo & Sitagliptin & Placebo \\
\hline $\begin{array}{l}\text { Background } \\
\text { medications }\end{array}$ & Metformin & Metformin + sulfonylurea & $\begin{array}{l}\text { Metformin }+ \\
\text { sulfonylurea }\end{array}$ & $\begin{array}{l}\text { Metformin }+ \\
\text { sitagliptin }\end{array}$ \\
\hline Study length & $\begin{array}{l}52 \text { weeks (26-week core and } \\
26 \text {-week extension) }\end{array}$ & $\begin{array}{l}52 \text { weeks (26-week core } \\
\text { and } 26 \text {-week extension) }\end{array}$ & 52 weeks & 26 weeks \\
\hline Regions & $\begin{array}{l}\text { North America, Europe, } \\
\text { Central/South America, } \\
\text { other regions }\end{array}$ & $\begin{array}{l}\text { North America, Europe, } \\
\text { Central America, other } \\
\text { regions }\end{array}$ & $\begin{array}{l}\text { North America, Europe, } \\
\text { Central/South America, } \\
\text { other regions }\end{array}$ & $\begin{array}{l}\text { North America, } \\
\text { Europe, Australia }\end{array}$ \\
\hline Patients, $n$ & 1284 & 469 & 755 & 213 \\
\hline Female, \% & 52.9 & 49.0 & 44.1 & 43.2 \\
\hline Age $^{\mathrm{a}}$, years & $55.4 \pm 9.4$ & $56.8 \pm 9.3$ & $56.7 \pm 9.5$ & $57.4 \pm 9.7$ \\
\hline $\mathrm{HbA}_{1 \mathrm{c}}{ }^{\mathrm{a}}, \%$ & $7.9 \pm 0.9$ & $8.1 \pm 0.9$ & $8.1 \pm 0.9$ & $8.5 \pm 0.8$ \\
\hline $\mathrm{FPG}^{\mathrm{a}}, \mathrm{mmol} / \mathrm{L}$ & $9.4 \pm 2.3$ & $9.5 \pm 2.2$ & $9.3 \pm 2.6$ & $10.2 \pm 2.3$ \\
\hline Body weight $^{\mathrm{a}}, \mathrm{kg}$ & $87.2 \pm 21.7$ & $92.8 \pm 22.4$ & $88.3 \pm 23.2$ & $92.1 \pm 20.7$ \\
\hline $\mathrm{BMI}^{\mathrm{a}}, \mathrm{kg} / \mathrm{m}^{2}$ & $31.8 \pm 6.2$ & $33.1 \pm 6.5$ & $31.6 \pm 6.9$ & $32.0 \pm 5.7$ \\
\hline $\begin{array}{l}\text { Duration of } \mathrm{T}^{2} \mathrm{DM}^{\mathrm{a}} \text {, } \\
\text { years }\end{array}$ & $6.9 \pm 5.3$ & $9.6 \pm 6.3$ & $9.6 \pm 6.2$ & $9.9 \pm 5.7$ \\
\hline $\begin{array}{l}\text { Patients included in } \\
\text { pooled PRO } \\
\text { analysis }\end{array}$ & 1193 & 432 & 704 & 207 \\
\hline \multicolumn{5}{|l|}{ Baseline PRO scores } \\
\hline \multicolumn{5}{|c|}{ CHES-Q satisfaction with weight } \\
\hline$n$ & 1193 & 432 & 704 & 207 \\
\hline Dissatisfied, $n(\%)$ & $859(72.0)$ & $343(79.4)$ & $471(66.9)$ & $151(72.9)$ \\
\hline Satisfied, $n(\%)$ & $334(28.0)$ & 89 (20.6) & $233(33.1)$ & $56(27.1)$ \\
\hline \multicolumn{5}{|c|}{ CHES-Q physical domain } \\
\hline$n$ & 1070 & 361 & 622 & 177 \\
\hline Median (IQR) & $4.17(3.33-5.33)$ & $4.17(3.00-5.17)$ & $4.33(3.50-5.33)$ & $4.17(3.17-5.17)$ \\
\hline \multicolumn{5}{|c|}{ CHES-Q emotional domain } \\
\hline$n$ & 1070 & 361 & 622 & 177 \\
\hline Median (IQR) & $5.67(4.33-6.00)$ & $5.67(4.33-6.00)$ & $5.67(4.67-6.00)$ & $5.33(4.33-6.00)$ \\
\hline \multicolumn{5}{|c|}{ IWQoL-Lite total score } \\
\hline$n$ & 1065 & 360 & 619 & NA \\
\hline Median (IQR) & $84.7(67.7-93.5)$ & $83.9(68.1-95.2)$ & $87.1(71.0-95.2)$ & - \\
\hline \multicolumn{5}{|l|}{ SF-36 MCS } \\
\hline$n$ & 1066 & 355 & 618 & NA \\
\hline Median (IQR) & $48.7(41.0-55.6)$ & $51.7(42.8-57.1)$ & $52.4(44.1-57.5)$ & - \\
\hline \multicolumn{5}{|l|}{ SF-36 PCS } \\
\hline$n$ & 1066 & 355 & 618 & NA \\
\hline Median (IQR) & $48.6(42.6-53.3)$ & $49.7(42.9-54.2)$ & $48.3(41.6-53.2)$ & - \\
\hline
\end{tabular}

$B M I$ body mass index, $C H E S-Q$ Current Health Satisfaction Questionnaire, $F P G$ fasting plasma glucose, $H b A_{l c}$ glycosylated hemoglobin, $I Q R$ interquartile range, IWQoL-Lite Impact of Weight on Quality of Life-Lite, MCS mental component summary score, PCS physical component summary score, $P R O$ patient-reported outcome, $S F-36$ Short Form-36, T2DM type 2 diabetes mellitus

${ }^{\mathrm{a}}$ Data are mean \pm standard deviation

${ }^{\mathrm{b}}$ Participants had to have baseline and follow-up PRO data to be included in the pooled analysis

which is not surprising given the differences in disease duration, region, and background of AHA medications across trials. This finding is consistent with clinical results using pooled data sets.

\subsection{Interest in Continuing Treatment}

At the end of each study (week 52 in Studies 1-3 and week 26 in Study 4), participants were more likely to express 
Table 3 Baseline demographic and clinical characteristics and PRO scores in the pooled population
Placebo/sitagliptin $(n=1102) \quad$ Canagliflozin $(n=1434)$

Baseline characteristics

Age, years

Mean (SD)

$56.2(9.3)$

$56.0(9.4)$

Sex, $n(\%)$

Female

528 (47.9)

$717(50.0)$

Male

$574(52.1)$

$717(50.0)$

Race, $n(\%)$

White

778 (70.6)

1017 (70.9)

Asian

$132(12.0)$

188 (13.1)

African American or Black

$82(7.4)$

$88(6.1)$

American Indian or Alaska Native

$13(1.2)$

$14(1.0)$

$3(0.2)$

$105(7.3)$

15 (1.0)

$1(0.1)$

$3(0.2)$

BMI, $\mathrm{kg} / \mathrm{m}^{2}$

Mean (SD)

$\mathrm{HbA}_{1 \mathrm{c}}, \%$

Mean (SD)

$71(6.4)$

18 (1.6)

$0(0.0)$

$4(0.4)$

$32.1(6.5)$

$31.8(6.3)$

$8.1(0.9)$

Systolic blood pressure, $\mathrm{mmHg}$

Mean (SD)

$8.0(0.9)$

$130(13.0)$

Diastolic blood pressure, $\mathrm{mmHg}$

Mean (SD)

129 (13.4)

$78.4(8.2)$

Study, $n(\%)$

Study 1

Study 2

Study 3

Study 4

$78.2(8.2)$

$691(48.2)$

502 (45.6)

291 (20.3)

141 (12.8)

349 (24.3)

355 (32.2)

$103(7.2)$

Treatment, $n(\%)$

Canagliflozin

104 (9.4)

$1434(100.0)$

Placebo

Sitagliptin

$0(0.0)$

408 (37.0)

$0(0.0)$

694 (63.0)

$0(0.0)$

Baseline PRO scores

CHES-Q satisfaction with weight

$n$

1102

Dissatisfied, $n(\%)$

795 (72.1)

1434

Satisfied, $n(\%)$

307 (27.9)

1029 (71.8)

405 (28.2)

CHES-Q physical domain

$n$

938

1292

Median (IQR)

Range

$4.33(3.33-5.33)$

$1-7$

$4.17(3.17-5.33)$

1-7

CHES-Q emotional domain

$n$

938

1292

Median (IQR)

Range

$5.67(4.33-6.00)$

$1-7$

$5.33(4.33-6.00)$

1-7

IWQoL-Lite total score

$n$

849

1195

Median (IQR)

$85.5(68.5-94.4)$

84.7 (68.5-94.4)

0-100

0-100 
Table 3 continued

\begin{tabular}{lll}
\hline & Placebo/sitagliptin $(n=1102)$ & Canagliflozin $(n=1434)$ \\
\hline SF-36 MCS & & \\
$n$ & 848 & 1191 \\
Median (IQR) & $50.9(43.0-57.1)$ & $49.6(41.6-56.2)$ \\
Range & $0-100$ & $0-100$ \\
SF-36 PCS & & \\
$n$ & 848 & 1191 \\
Median (IQR) & $48.3(42.2-53.2)$ & $48.8(42.3-53.6)$ \\
Range & $0-100$ & $0-100$
\end{tabular}

$B M I$ body mass index, CHES- $Q$ Current Health Satisfaction Questionnaire, $H b A_{1 c}$ glycosylated hemoglobin, IQR interquartile range, IWQoL-Lite Impact of Weight on Quality of Life-Lite, MCS mental component summary score, $P C S$ physical component summary score, $P R O$ patient-reported outcome, $S D$ standard deviation, SF-36 Short Form-36

interest in continuing treatment with canagliflozin compared with placebo/sitagliptin [OR $(95 \% \mathrm{CI})$ of 1.54 (1.19-1.99); $p=0.001$ ], with 85.6 and $79.8 \%$ of participants expressing interest in continuing each treatment, respectively.

\subsection{Effects of Canagliflozin on Health-Related Quality of Life}

\subsubsection{Weight Satisfaction and Impact of Weight on Quality of Life}

Responses to the CHES-Q item 1 in Studies 1-4 indicated that participants treated with canagliflozin were more likely to be satisfied with their body weight after 26 weeks compared with placebo/sitagliptin [OR $(95 \% \mathrm{CI})$ of 1.44 $(1.20-1.74) ; p<0.001$; Fig. 1 ), with 68.0 and $61.8 \%$ of participants responding favorably, respectively.

Canagliflozin treatment was also associated with statistically significant improvements in IWQoL-Lite total scores compared with placebo/sitagliptin in Studies 1-3 [LS mean change from baseline of 3.33 and 1.96, respectively; difference (95\% CI) of $1.37(0.37-2.37) ; p=0.007$; Fig. 2a]; however, the magnitude of the average change did not reach the threshold for MCID in either group. Importantly, participants treated with canagliflozin were more likely to have a favorable outcome for IWQoL-Lite total score compared with placebo/sitagliptin (OR [95\% CI] of 1.26 [1.05, 1.51]; $p=0.012$; Fig. 1), with 51.3 and $45.2 \%$ of participants responding favorably, respectively.

\subsubsection{Physical Satisfaction and Function}

Responses to the CHES-Q physical domain in Studies 1-4 indicated that participants treated with canagliflozin had significantly greater improvements in physical health satisfaction scores compared with placebo/sitagliptin at week
26 [LS mean change from baseline of 0.47 and 0.29 , respectively; difference (95\% CI) of 0.18 (0.10-0.26); $p<0.001$; Fig. 2b], which reached the threshold of MCID. Participants who received canagliflozin were more likely to have maintained or improved satisfaction with physical health than those who received placebo/sitagliptin [OR (95\% CI) of 1.23 (1.03-1.48); $p=0.026$; Fig. 1]; 76.5 and $72.7 \%$ of participants treated with canagliflozin and placebo/sitagliptin, respectively, maintained or improved satisfaction with their physical health.

In Studies 1-3, an improvement in SF-36 physical component scores was seen with canagliflozin, whereas a decline was seen with placebo/sitagliptin [LS mean changes of 0.52 and -0.17 , respectively; difference $(95 \% \mathrm{CI})$ of $0.69(0.17-1.21) ; p=0.009$; Fig. 2b]. Although statistically significant, the magnitude of difference was not enough to reach the MCID over 26 weeks. Favorable outcomes in SF-36 physical component summary scores were reported by 45.7 and $44.5 \%$ of participants treated with canagliflozin and placebo/sitagliptin, respectively [OR (95\% CI) of $1.07(0.89-1.30) ; p=0.457$; Fig. 1].

\subsubsection{Emotional Health Satisfaction and Mental Function}

Responses to the CHES-Q emotional domain in Studies 1-4 demonstrated small but significantly greater improvements in emotional health satisfaction score with canagliflozin compared with placebo/sitagliptin at week 26 [LS mean changes of 0.22 and 0.07 , respectively; difference $(95 \% \mathrm{CI})$ of $0.15(0.06-0.23) ; p=0.001$; Fig. 2b]; however, the magnitude of improvement was not enough to reach the threshold for MCID. Participants were also more likely to maintain a good score or improve their emotional health satisfaction score with canagliflozin vs. placebo/ sitagliptin [OR (95\% CI) of 1.27 (1.02-1.57); $p=0.032$; Fig. 1], with 81.7 and $78.9 \%$ of participants reporting favorable outcomes, respectively. 


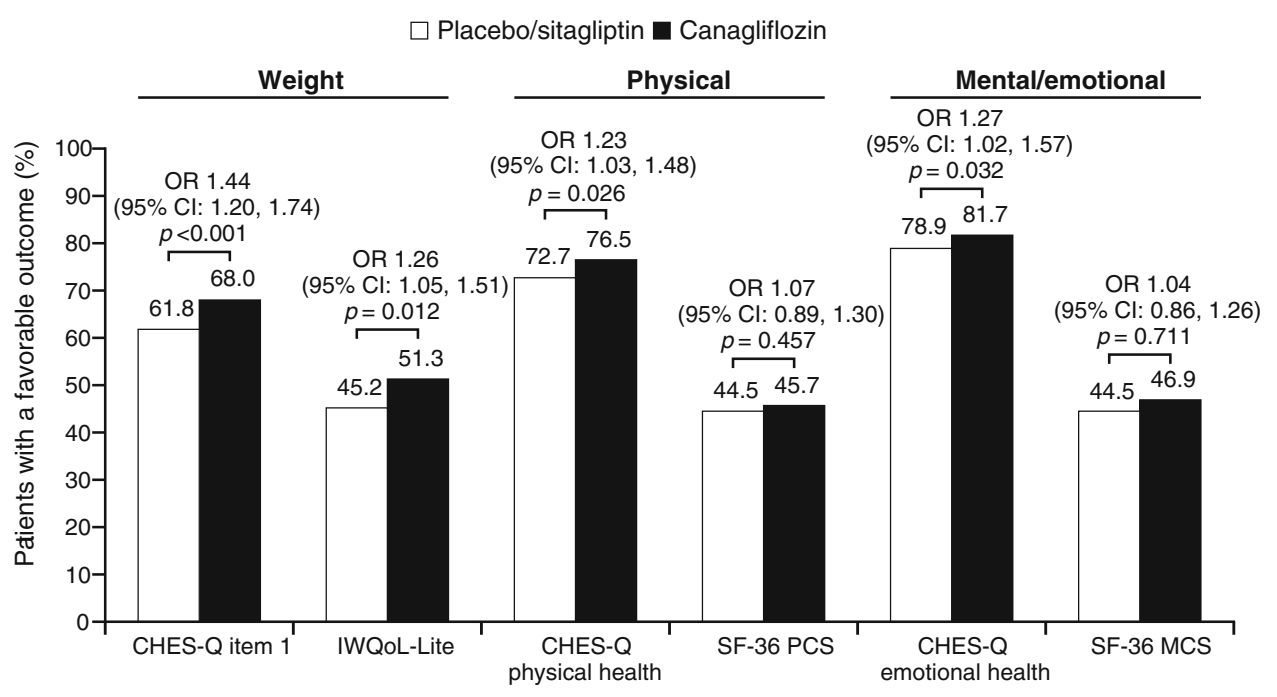

Fig. 1 Proportion of participants with favorable outcomes of patientreported outcome measures at week 26. ${ }^{\text {a }}$ CHES- $Q$ Current Health Satisfaction Questionnaire, CI confidence interval, IWQoL-Lite Impact of Weight on Quality of Life-Lite, MCS mental component summary score, $O R$ odds ratio, $P C S$ physical component summary

Changes in SF-36 mental component scores in Studies 1-3 were similar with canagliflozin and placebo/sitagliptin at week 26 [LS mean changes of 0.42 and 0.33 , respectively; difference $(95 \% \mathrm{CI})$ of $0.09(-0.59$ to 0.77$)$; $p=0.787$; Fig. 2b]. Favorable outcomes in SF-36 mental component summary scores were reported by 46.9 and $44.5 \%$ of participants treated with canagliflozin and placebo/sitagliptin, respectively [OR $(95 \% \mathrm{CI})$ of 1.04 (0.86-1.26); $p=0.711$; Fig. 1].

\subsection{Sensitivity Analyses}

Results from sensitivity analyses adjusted for covariates including sex and baseline body mass index were generally similar to the primary and secondary analyses (Tables 2 and 3 of the ESM). Although the sample size was smaller for the sensitivity analysis of data at week 52, results were consistent with those seen at week 26 for interest in continuing study medication with canagliflozin (Table 4 of the ESM); improvements in satisfaction with weight based on CHES-Q item 1 and the CHES-Q physical domain were also seen with canagliflozin vs. placebo/sitagliptin. Improvement in SF-36 physical and mental component summary scores were also consistent with week 26 results, though treatment differences were attenuated for IWQoLLite. At week 52, the LS mean difference between treatment groups was statistically significant for the CHES-Q physical domain, but not for the SF-36 physical component summary score.

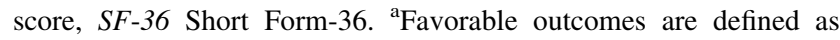
maintaining high baseline scores through the follow-up period or improvement at follow-up for participants with lower baseline scores (refer to Table 1 for more details)

\section{Discussion}

This pooled analysis found that a significantly higher proportion of participants treated with canagliflozin were interested in continuing treatment at the end of the study compared with placebo/sitagliptin, implying the potential for better real-world medication adherence with canagliflozin. Adherence to medication is necessary for people with T2DM to experience the benefits of treatment [26] and is one of the seven self-care behaviors for ideal management of diabetes [27]. Overall, medication adherence in clinical practice is suboptimal, with nearly one quarter of patients with T2DM non-adherent to their oral AHA regimen [28]. Improved adherence to medication can lead to improved glycemic control and is associated with performance of other healthy behaviors, such as healthy eating, being active, and weight management, which are essential for successful and effective self-management of diabetes [29]. Findings from a retrospective study, which showed that real-world medication adherence rates were higher among those receiving canagliflozin compared with other newer oral and injectable AHAs, support the idea that interest in continuing treatment can lead to better adherence over time [30]. Although results from this pooled population suggest a willingness to continue treatment with canagliflozin and a potential for improved adherence to medication, the attitudes and behaviors of these study participants may not be representative of patients with T2DM outside of clinical trials.

Findings from this pooled analysis also demonstrated some statistically significant, but not necessarily clinically 
Fig. 2 Change from baseline in patient-reported outcomes related to (a) weight and (b) physical and mental/ emotional satisfaction. $C H E S-Q$ Current Health Satisfaction Questionnaire, $C I$ confidence interval, IWQoL-Lite Impact of Weight on Quality of Life-Lite, LSM least squares mean, $M C S$ mental component summary score, $P C S$ physical component summary score, $S F-36$ Short Form-36
(A)

Placebo/sitagliptin @ Canagliflozin

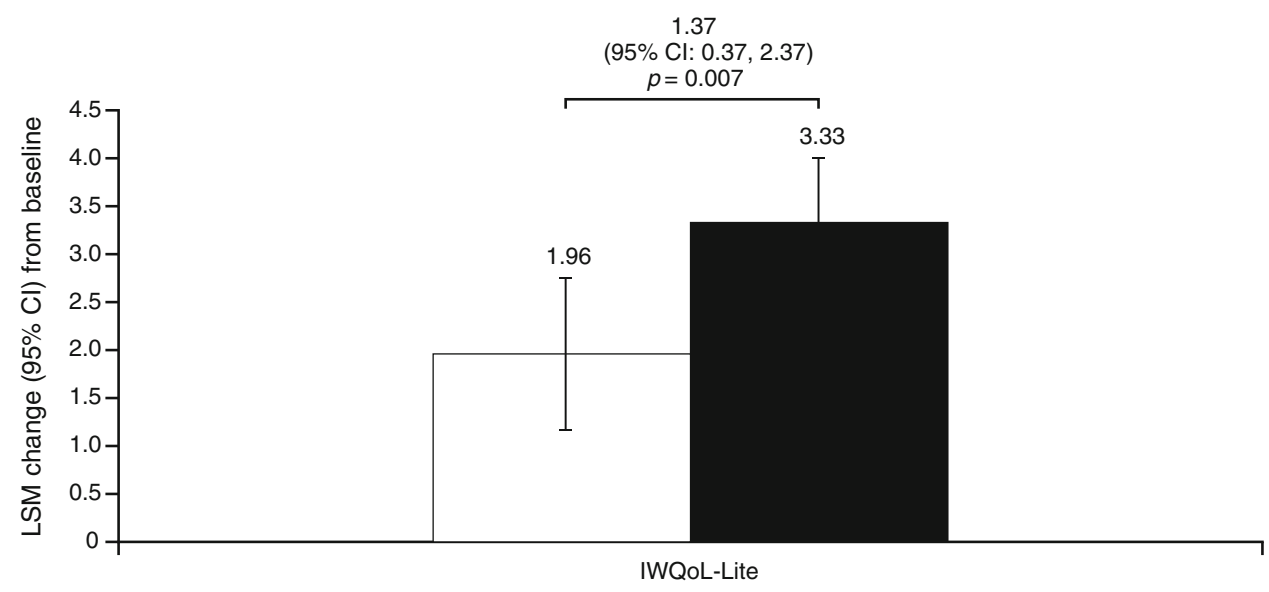

$\square$ Placebo/sitagliptin $\square$ Canagliflozin

(B)

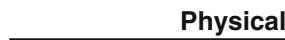

Mental/emotional

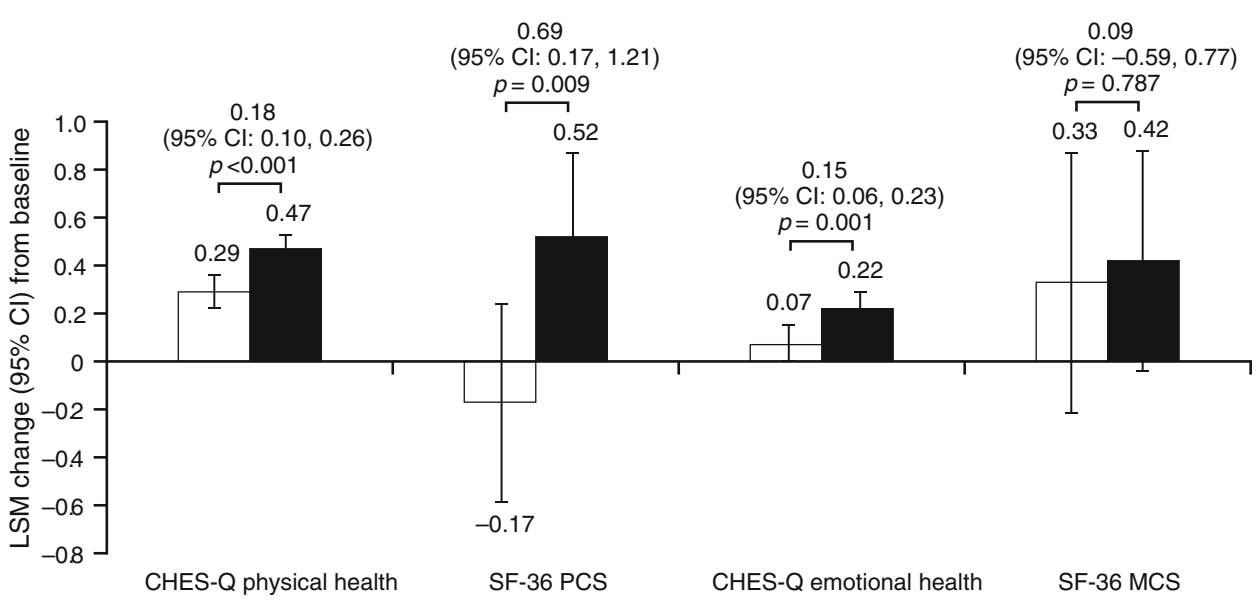

significant, improvements in HRQoL with canagliflozin compared with placebo or sitagliptin. Satisfaction with weight was significantly higher with canagliflozin compared with placebo or sitagliptin, which is not surprising given the known weight-loss effects of canagliflozin. Previous studies have established that the amount of weight loss experienced with canagliflozin meaningfully improves weight-related quality of life and satisfaction with physical and emotional health [19], and that people who lost weight were more likely to engage in other healthy behaviors several months after the start of treatment, including taking action to lose additional weight, following a healthy diet, and exercising [7]. IWQoL-Lite was used in this study because it is a weight-specific measure, and while the improvement in the IWQoL-Lite total score did not reach the level of clinical significance, a higher proportion of participants experienced a favorable outcome (improvement of an unfavorable score or maintenance of a good score) following treatment with canagliflozin vs. placebo or sitagliptin. Generally, people with higher baseline PRO scores are less likely to show improvement than patients with low baseline scores, a phenomenon known as the ceiling effect [31]. Participants in this pooled analysis had relatively high baseline scores, which may have resulted in smaller observed average improvements in PRO scores.

In addition to weight-related quality of life, statistically significant improvements were seen for all measures of physical function and for the disease-specific measure of emotional function. Small changes that did not approach the level of clinical significance were observed in SF-36 scores. These results are not unexpected because, unlike CHES-Q, the SF-36 is not a T2DM- or weight-specific instrument and thus is not a very sensitive measure for these participants. No difference was observed in the mental quality-of-life measurement, consistent with previous reports of people with T2DM [31]. Findings from the 
sensitivity analyses were generally consistent with the results from the main analyses, indicating that sex and baseline body mass index did not have a significant effect on PROs.

The improvements in HRQoL with canagliflozin that were observed in this analysis may be partly driven by the recognizable benefits of treatment, including weight loss [7, 19]. Most patients with T2DM treated with canagliflozin experience rapid weight loss over the first 6 weeks of treatment, followed by slower weight loss, and a plateau after 34 weeks [14]. Favorable weight loss-related effects on HRQoL outcomes have been demonstrated in other studies in patients with T2DM. After the first year of the Look AHEAD (Action for Health in Diabetes) trial, patients who lost weight while receiving intensive lifestyle intervention had improved physical fitness and reduced physical symptoms [31]. Over 8 years in the study, patients who received lifestyle intervention and lost weight maintained better physical function and were less likely to experience symptoms of depression [32]. Similarly, improvements were observed in HRQoL among patients who lost weight while receiving intensive lifestyle intervention in the Diabetes Prevention Program [33]. Further research is needed to explore the relationship between PROs and weight loss in patients treated with canagliflozin.

Previous analyses of canagliflozin data have shown that weight loss of $\sim 2-3 \mathrm{~kg}$ is associated with improvements in HRQoL [19], implying that this amount of weight loss is noticeable to patients. While canagliflozin has demonstrated significantly greater mean weight reductions vs. comparators, about a third of patients randomized to placebo or sitagliptin lost some weight during the trials as a result of background diet and exercise. The diabetes education and frequent monitoring within these trials are likely responsible for some of this weight loss, as structured programs have been shown to help people with T2DM achieve weight loss in the real world [34]. Because weight loss appears to drive PRO benefits, it may be expected that participants who lost weight may experience HRQoL improvements regardless of treatment assignment.

There were several limitations of this study. This study relied on pooling of separate studies with differences in participant characteristics and background AHA medication use, which may have reduced the observable effects of canagliflozin treatment on PROs. Additionally, the use of MCID as a threshold for determining whether changes were clinically meaningful may limit the interpretation of this study, as MCIDs are not well defined for some PRO instruments, including IWQoL-Lite and SF-36 [19, 24, 25, 35]. Additionally, medication adherence was not measured in these studies, which would have allowed for assessment of whether interest in continuing study medication correlates with improved adherence. However, the controlled nature of randomized trials may not be conducive to capturing estimates of medication adherence comparable to those in real-world settings.

Further analyses correlating patients' experience with weight change and willingness to continue treatment at intermediate time points might also be informative, as previous work has shown that a patient's experience with weight over time can impact PRO responses [7]. In addition, it would be informative to determine how these findings translate to real-world clinical settings, especially related to adherence to medication and healthy behaviors.

Analysis of PRO endpoints is a challenge owing to the variability of responses across patients; as a result, clinical trials in T2DM do not always consider power for PRO endpoints in sample size calculations. Therefore, pooled analyses are useful for increasing statistical power to detect treatment effects. This analysis was strengthened by pooling data from individual participants from several trials of canagliflozin, which provided sufficient statistical power to reveal a treatment effect for canagliflozin vs. placebo/sitagliptin. It is unknown whether improvements in satisfaction and HRQoL or expressing interest in continuing medication actually result in improved medication adherence in real-world settings.

\section{Conclusion}

In summary, findings from this analysis suggest that people receiving canagliflozin have a positive experience with their treatment and report meaningful outcomes in addition to the traditional target of glycemic control. Importantly, participants expressed interest in continuing study medication, which together with improvements in overall health satisfaction and physical and emotional health, may be important for patient-centered disease management and treatment adherence.

Acknowledgements Medical writing support was provided by Dana Tabor, Ph.D., of MedErgy, and was funded by Janssen Scientific Affairs, LLC and Janssen Global Services, LLC.

Author Contributions JC, LMD, and ST contributed to the conception and design of this study, and data interpretation. SA and AS contributed to the data analysis and interpretation. All authors participated in the drafting and revision of this manuscript, approved it in its final form, and guarantee the overall content.

\section{Compliance with Ethical Standards}

Funding This analysis was supported by Janssen Scientific Affairs, LLC and Janssen Global Services, LLC, using data from studies funded by Janssen Research \& Development, LLC. Canagliflozin has been developed by Janssen Research \& Development, LLC, in collaboration with Mitsubishi Tanabe Pharma Corporation. 
Conflict of interest Jennifer Cai is a full-time employee of Janssen Scientific Affairs, LLC. Linda M. Delahanty is a consultant for Janssen Global Services, LLC. Sydney Akapame and April Slee are full-time employees of Axio Research, and they received payment from Janssen for statistical support of the analyses reported in this manuscript. Shana Traina is a full-time employee of Janssen Global Services, LLC.

Ethics approval All procedures performed in studies involving human participants were in accordance with the ethical standards of the institutional and/or national research committee and with the 1964 Helsinki Declaration and its later amendments or comparable ethical standards.

Consent to participate Informed consent was obtained from all individual participants for the studies on which these analyses are based.

Open Access This article is distributed under the terms of the Creative Commons Attribution-NonCommercial 4.0 International License (http://creativecommons.org/licenses/by-nc/4.0/), which permits any noncommercial use, distribution, and reproduction in any medium, provided you give appropriate credit to the original author(s) and the source, provide a link to the Creative Commons license, and indicate if changes were made.

\section{References}

1. Inzucchi SE, Bergenstal RM, Buse JB, Diamant M, Ferrannini E, Nauck M, et al. Management of hyperglycemia in type 2 diabetes, 2015: a patient-centered approach: update to a position statement of the American Diabetes Association and the European Association for the Study of Diabetes. Diabetes Care. 2015;38(1):140-9.

2. Berwick DM, Nolan TW, Whittington J. The triple aim: care, health, and cost. Health Aff (Millwood). 2008;27(3):759-69.

3. American Diabetes Association. Standards of medical care in diabetes: 2017. Diabetes Care. 2017;40(Suppl. 1):S1-135.

4. Traina S, Slee A. Demystifying, "patient-centered" care in type 2 diabetes: the role of systematic measurement. Am J Manag Care. 2016;22(Special Issue 4):SP135-6.

5. US Food and Drug Administration. Public workshop: diabetes outcome measures beyond hemoglobin A1c (HbAlc). http:// www.fda.gov/Drugs/NewsEvents/ucm499281.htm. Accessed 21 Nov 2017.

6. US Department of Health and Human Services Food and Drug Administration. Guidance for industry: patient-reported outcome measures: use in medical product development to support labelling claims; 2009. http://www.fda.gov/downloads/Drugs/ GuidanceComplianceRegulatoryInformation/Guidances/UCM1932 82.pdf. Accessed 21 Nov 2017.

7. Traina SB, Slee A, Woo S, Canovatchel W. The importance of weight change experiences for performance of diabetes self-care: a patient-centered approach to evaluating clinical outcomes in type 2 diabetes. Diabetes Ther. 2015;6(4):611-25.

8. Trikkalinou A, Papazafiropoulou AK, Melidonis A. Type 2 diabetes and quality of life. World J Diabetes. 2017;8(4):120-9.

9. Correa K, Gouvea GR, Silva MA, Possobon RF, Barbosa LF, Pereira AC, et al. Quality of life and characteristics of diabetic patients. Cien Saude Colet. 2017;22(3):921-30.

10. Hibbard JH, Stockard J, Mahoney ER, Tusler M. Development of the Patient Activation Measure (PAM): conceptualizing and measuring activation in patients and consumers. Health Serv Res. 2004;39(4 Pt 1):1005-26.

11. Rosenthal N, Meininger G, Ways K, Polidori D, Desai M, Qiu R, et al. Canagliflozin: a sodium glucose co-transporter 2 inhibitor for the treatment of type 2 diabetes mellitus. Ann NY Acad Sci. 2015;1358(1):28-43.

12. Thayer S, Aguilar R, Korrer S, Chow W. HbA1c outcomes in patients treated with canagliflozin versus sitagliptin in US health plans. Clin Ther. 2017;39(10):2061-72.

13. Thayer S, Chow W, Korrer S, Aguilar R. Real-world evaluation of glycemic control among patients with type 2 diabetes mellitus treated with canagliflozin versus dipeptidyl peptidase-4 inhibitors. Curr Med Res Opin. 2016;32(6):1087-96.

14. Lavalle-González FJ, Januszewicz A, Davidson J, Tong C, Qiu R, Canovatchel W, et al. Efficacy and safety of canagliflozin compared with placebo and sitagliptin in patients with type 2 diabetes on background metformin monotherapy: a randomised trial. Diabetologia. 2013;56(12):2582-92.

15. Wilding JP, Charpentier G, Hollander P, Gonzalez-Galvez G, Mathieu C, Vercruysse F, et al. Efficacy and safety of canagliflozin in patients with type 2 diabetes mellitus inadequately controlled with metformin and sulphonylurea: a randomised trial. Int J Clin Pract. 2013;67(12):1267-82.

16. Schernthaner G, Gross JL, Rosenstock J, Guarisco M, Fu M, Yee $\mathrm{J}$, et al. Canagliflozin compared with sitagliptin for patients with type 2 diabetes who do not have adequate glycemic control with metformin plus sulfonylurea: a 52-week, randomized trial. Diabetes Care. 2013;36(9):2508-15.

17. Rodbard HW, Seufert J, Aggarwal N, Cao A, Fung A, Pfeifer M, et al. Efficacy and safety of titrated canagliflozin in patients with type 2 diabetes mellitus inadequately controlled on metformin and sitagliptin. Diabetes Obes Metab. 2016;18(8):812-9.

18. Cefalu WT, Leiter LA, Yoon KH, Arias P, Niskanen L, Xie J, et al. Efficacy and safety of canagliflozin versus glimepiride in patients with type 2 diabetes inadequately controlled with metformin (CANTATA-SU): 52 week results from a randomised, double-blind, phase 3 non-inferiority trial. Lancet. 2013;382(9896):941-50.

19. Traina S, Guthrie R, Slee A. The impact of weight loss on weightrelated quality of life and health satisfaction: results from a trial comparing canagliflozin with sitagliptin in triple therapy among people with type 2 diabetes. Postgrad Med. 2014;126(3):7-15.

20. Traina SB, Colwell HH, Crosby RD, Mathias SD. Pragmatic measurement of health satisfaction in people with type 2 diabetes mellitus using the Current Health Satisfaction Questionnaire. Patient Relat Outcome Meas. 2015;6:103-15.

21. Traina SB, McQuarrie K, Barrett A, DiBenedetti D, McLeod L. Content validity of the Current Health Satisfaction Questionnaire (CHES-Q) among people living with type 2 diabetes mellitus (T2DM) and comorbid chronic kidney disease (CKD). Value Health. 2015;18(7):A710-1.

22. Janssen Global Services LLC. Current Health Satisfaction Questionnaire (CHES-Q): scoring documentation. 2015.

23. Kolotkin RL, Crosby RD, Kosloski KD, Williams GR. Development of a brief measure to assess quality of life in obesity. Obes Res. 2001;9(2):102-11.

24. Crosby RD, Kolotkin RL, Williams GR. An integrated method to determine meaningful changes in health-related quality of life. J Clin Epidemiol. 2004;57(11):1153-60.

25. Bjorner JB, Lyng Wolden M, Gundgaard J, Miller KA. Benchmarks for interpretation of score differences on the SF-36 health survey for patients with diabetes. Value Health. 2013;16(6):993-1000.

26. Jermendy G, Wittmann I, Nagy L, Kiss Z, Rokszin G, BonyiToth $Z$, et al. Persistence of initial oral antidiabetic treatment in 
patients with type 2 diabetes mellitus. Med Sci Monit. 2012;18(2):CR72-7.

27. American Association of Diabetes. Educators position statement. AADE7 self-care behaviors. 2014.

28. Iglay K, Cartier SE, Rosen VM, Zarotsky V, Rajpathak SN, Radican L, et al. Meta-analysis of studies examining medication adherence, persistence, and discontinuation of oral antihyperglycemic agents in type 2 diabetes. Curr Med Res Opin. 2015;31(7):1283-96.

29. Hernandez-Tejada MA, Campbell JA, Walker RJ, Smalls BL, Davis KS, Egede LE. Diabetes empowerment, medication adherence and self-care behaviors in adults with type 2 diabetes. Diabetes Technol Ther. 2012;14(7):630-4.

30. Cai J, Wang Y, Baser O, Xie L, Chow W. Comparative persistence and adherence with newer anti-hyperglycemic agents to treat patients with type 2 diabetes in the United States. J Med Econ. 2016;19(12):1175-86.

31. Williamson DA, Rejeski J, Lang W, Van Dorsten B, Fabricatore $\mathrm{AN}$, Toledo $\mathrm{K}$, et al. Impact of a weight management program on health-related quality of life in overweight adults with type 2 diabetes. Arch Intern Med. 2009;169(2):163-71.

32. Rubin RR, Wadden TA, Bahnson JL, Blackburn GL, Brancati FL, Bray GA, et al. Impact of intensive lifestyle intervention on depression and health-related quality of life in type 2 diabetes: the Look AHEAD Trial. Diabetes Care. 2014;37(6):1544-53.

33. Florez H, Pan Q, Ackermann RT, Marrero DG, Barrett-Connor E, Delahanty L, et al. Impact of lifestyle intervention and metformin on health-related quality of life: the diabetes prevention program randomized trial. J Gen Intern Med. 2012;27(12):1594-601.

34. Ali MK, Echouffo-Tcheugui J, Williamson DF. How effective were lifestyle interventions in real-world settings that were modeled on the Diabetes Prevention Program? Health Aff (Millwood). 2012;31(1):67-75.

35. Maruish ME. User's manual for the SF-36v2 health survey. 3rd ed. Lincoln: QualityMetric, Inc.; 2011. 